\title{
Studiengänge zur Versorgungsforschung in Deutschland: aktueller Stand und zukünftige Perspektiven
}

\section{Educational Programmes in Health Services Research in Germany: Current State and Future Perspectives}

(ㄷ)(1) $(9)$

Autoren

Silke Kuske' ${ }^{1}$, Christine Holmberg ${ }^{2}$ (D) ${ }^{3}$, Michel Wensing ${ }^{4}$ (D), Bernd Reuschenbach ${ }^{5}$, Andreas Büscher ${ }^{6}$ D,

Edmund Neugebauer ${ }^{7}$, Holger Pfaff ${ }^{8}$ D, Ute Karbach ${ }^{9}$, Katrin Balzer ${ }^{10}$, Sascha Köpke ${ }^{11}$ D, Nicole Ernstmann ${ }^{12}$

Institute

1 Pflegewissenschaft und Versorgungsforschung, Fliedner Fachhochschule Düsseldorf, Düsseldorf

2 Institut für Sozialmedizin und Epidemiologie, Medizinische Hochschule Brandenburg Theodor Fontane, Brandenburg an der Havel

3 Fakultät für Gesundheitswissenschaften Brandenburg, Medizinische Hochschule Brandenburg Theodor Fontane, Neuruppin

4 Abteilung Allgemeinmedizin und Versorgungsforschung, Universitätsklinikum Heidelberg, Heidelberg

5 Fakultät für Gesundheit und Pflege, Katholische Stiftungshochschule München, München

6 Fakultät Wirtschafts- und Sozialwissenschaften, Deutsches Netzwerk für Qualitätsentwicklung in der Pflege (DNQP), Hochschule Osnabrück, Osnabrück

7 Medizinische Hochschule Brandenburg (MHB), Neuruppin

8 Institut für Medizinsoziologie, Versorgungsforschung und Rehabilitationswissenschaft, Zentrum für Versorgungsforschung, Universität zu Köln, Köln

9 Fakultät Rehabilitationswissenschaften, Technische Universität Dortmund, Dortmund

10 Sektion für Forschung und Lehre in der Pflege, Institut für Sozialmedizin und Epidemiologie, Universität zu Lübeck, Lübeck

11 Instiut für Pflegewissenschaft, Medizinische Fakultät und Uniklinik Köln, Universität zu Köln, Köln

12 Forschungsstelle für Gesundheitskommunikation und Versorgungsforschung (CHSR), Klinik und Poliklinik für Psychosomatische Medizin und Psychotherapie, Universitätsklinikum Bonn, Institut für Patientensicherheit, Rheinische Friedrich-Wilhelms-Universität Bonn, Bonn

Schlüsselwörter

Versorgungsforschung, Hochschulbildung, Ausbildungsforschung, Lehr- und Lernziele, Kerncurriculum, wissenschaftlicher Nachwuchs

\section{Key words}

health care services research, academic education, educational research, study aims, core curriculum, novice researchers online publiziert $\quad 10.12 .2020$

Bibliografie

Gesundheitswesen 2022; 84: 227-241

DOI 10.1055/a-1276-0686

ISSN $0941-3790$

(C) 2020. The Author(s).

This is an open access article published by Thieme under the terms of the Creative Commons Attribution-NonDerivative-NonCommercial-License, permitting copying and reproduction so long as the original work is given appropriate credit. Contents may not be used for commercial purposes, or adapted, remixed, transformed or built upon. (https://creativecommons. org/licenses/by-nc-nd/4.0/)

Georg Thieme Verlag KG, Rüdigerstraße 14,

70469 Stuttgart, Germany

Korrespondenzadresse

Prof. Silke Kuske

Pflegewissenschaft und Versorgungsforschung

Fliedner Fachhochschule Düsseldorf

Geschwister-Aufricht-Straße 9

40489 Düsseldorf

Deutschland

kuske@fliedner-fachhochschule.de

\section{ZUSAMMENFASSUNG}

Ziel der Studie Ziel dieser Untersuchung war es, die derzeit akkreditierten Versorgungsforschungsstudiengänge in Deutschland im Hinblick auf ihre Strukturdaten und Inhalte zu analysieren.

Methodik Im Rahmen eines deskriptiven qualitativen Designs wurden die aktuellen Studienverlaufspläne, die Modulhandbücher und die Steckbriefe akkreditierter Masterstudiengänge der Versorgungsforschung inhaltsanalytisch ausgewertet.

Ergebnisse Die 6 analysierten Studiengänge zeigen sich hinsichtlich ihrer strukturellen Merkmale und lehr-lerninhaltlichen Ausrichtung in den wesentlichen Punkten als homogen. Ziel aller Studiengänge ist es, fach- und disziplinübergreifende personale und soziale Kompetenzen zu fördern, um Versorgungsbedarfe im Gesundheitswesen zu identifizieren, Innovationen der Versorgung zu entwickeln, zu initiieren, zu evaluieren und kritisch zu reflektieren. 
Schlussfolgerungen Trotz ähnlicher Lehr-Lerninhalte bestehen spezifische Ausrichtungen, für die sich die Studierenden vor dem Hintergrund ihrer individuellen Präferenzen entscheiden können. Diese Ergebnisse könnten unter Berücksichtigung aktueller Diskurse und weiterer Erkenntnisse als Grundlage für Empfehlungen zur Entwicklung eines Kerncurriculums dienen.

\section{ABSTRACT}

Objective The aim of this study was to analyse accredited study programmes of health services research in Germany at Master's degree level regarding their structural data and content.
Methods Using a descriptive qualitative design, all included plans of study courses, module handbooks and descriptions of the Master's degree programmes were analysed.

Results The Master's degree programmes were similar in their structural elements as well as in their content of education. They aim at enhancing competences related to the areas of health services research to identify health care needs and to develop, initiate, evaluate and critically assess innovative care strategies across disciplines.

Discussion Although the content is similar, the study programmes focus on specific aspects that allow students to choose a certain programme depending on their preferences. A next step can be the development of a core curriculum that takes into consideration further findings and the discourse of different stakeholders.

\section{Einleitung}

Die Versorgungsforschung hat in Deutschland in den letzten 2 Jahrzehnten zunehmend durch verschiedene Förderinitiativen an Bedeutung gewonnen [1]. Als interdisziplinärer Ansatz verfolgt sie das Ziel, die Alltagswirksamkeit und die Qualität der Versorgung im Gesundheitswesen zu untersuchen und zu verbessern [2, 3].

2017 wurde im Rahmen einer Hochschullehrerbefragung eine erste Untersuchung der Versorgungsforschungsstrukturen auf hochschulischer Ebene vorgenommen [4]. Nur wenige Studiengänge der Versorgungsforschung konnten identifiziert werden, meist richteten sie sich auf die klinischen Versorgungsforschungsgebiete und bestanden aus Zusammenschlüssen aus Versorgungsforschung und einem weiteren theoretischen Fach. Nur eine für besondere Methodenfragen ausgerichtete Professur konnte beschrieben werden [4]. Zum Zeitpunkt der Erhebung wurden 3 akkreditierte Studiengänge der Versorgungsforschung an den Standorten Heidelberg, Köln und München identifiziert. Eine Empfehlung hinsichtlich des Ausbaus der Bildungsstrukturen in der Versorgungsforschung wurde aus den Ergebnissen abgeleitet [4]. In der Zwischenzeit sind weitere Studiengänge an den Standorten Lübeck, Osnabrück und Düsseldorf hinzugekommen. Mit dieser Entwicklung gewinnt der im Positionspapier der Gruppe der Hochschullehrer im Deutschen Netzwerk Versorgungsforschung (DNVF) formulierte Wunsch eines Orientierungsrahmens für ein Kerncurriculum für die Versorgungsforschung [5] zunehmend an Bedeutung. Als Grundlage für die Gegenstands- und Begriffsbestimmung der Versorgungforschung, für die Festlegung ihrer Anwendungsfelder, Aufgaben und Ziele sowie benötigten wissenschaftstheoretischen Grundannahmen, Modelle, Theorien, Studiendesigns, Datenquellen, Datenerhebungs- und Auswertungsmethoden können Stellungnahmen, Memoranden [6-12] und Lehrbuchbeiträge der Versorgungsforschung [2] herangezogen werden. Bislang ist wenig darüber bekannt, wie die Studiengänge der Versorgungsforschung strukturell ausgerichtet und welche der für Deutschland beschriebenen Gegenstandsbereiche und Methoden der Versorgungsforschung standortübergreifend tatsächlich abgebildet sind. Ziel dieser Untersuchung ist es daher, die derzeit akkreditierten Versorgungsforschungsstudiengänge in Deutschland zu beschreiben, diese im Hinblick auf ihre gemeinsamen und besonderen Strukturdaten und Inhalte zu analysieren sowie darauf basierend eine Diskussionsgrundlage für ein konsentiertes Kerncurriculum zu schaffen.

\section{Methodik}

Auf Basis eines qualitativen Designs wurden die Studienverlaufspläne, die Modulhandbücher und die auf der DNVF Webseite - Gruppe der Hochschullehrer - veröffentlichten Steckbriefe akkreditierter Masterstudiengänge (davon 5 veröffentlicht am 18.09.2019, einer am 02.07.2020) der Versorgungsforschung inhaltsanalytisch ausgewertet. Die Daten wurden extrahiert und primär induktiv analysiert [13]. Eingeschlossen wurden Studiengänge der Versorgungsforschung, die bis einschließlich des Sommersemesters 2020 akkreditiert wurden. Die Daten stammten aus den Studienverlaufsplänen, aus den Modulhandbüchern selbst und den Studiensteckbriefen. Alle Modulhandbücher in der letztgültigen Fassung vom 21.05.2017 (1), 21.06.2017 (2), 14.02.2019(3), 24.06.2019 (4), 23.01.2019(5) und dem 30.03.2019 (6), wurden durch die jeweiligen Studiengangsvertretungen zur Verfügung gestellt.

Zunächst wurden die Daten der Studienverlaufspläne als Kodiereinheit extrahiert (Bezeichnungen der Module als höchste Abstraktionsebene), um eine erste Grundorientierung zu erhalten. Die Lehr-Lerninhalte der Modulhandbücher wurden in insgesamt 3 Vorgängen entlang den jeweils dort angegeben Abstraktionsebenen (Überschriften, Subüberschriften usw.) zunächst extrahiert, um ihre individuellen Ordnungslogiken nachzuvollziehen. Die Analyserichtung erfolgte von abstrakter zur konkreten Ebene. Im zweiten Schritt wurden die Daten durch die Analyse der Modulbeschreibungen angereichert und ein induktives Kategoriengerüst ausgearbeitet. Hierzu wurden die Lehr-Lerninhaltsebenen und nicht die dort angegeben Kompetenzebenen analysiert. Die Terminologie in den jeweiligen Modulhandbüchern wurde weitestgehend übernommen, jedoch im Laufe der verschiedenen Analysedurchgänge durch eigene Kategorien erweitert, um divergente Bezeichnungen gleichen Inhalts auf höherer Abstraktionsebene zu kategorisieren. Diese Kategorien wurden durch das Zeichen „ * “ in > Tab. 3 kenntlich gemacht. Im letzten Schritt wurde das auf höchster Abstrak- 
- Tab. 1 Strukturdaten der Studiengänge der Versorgungsforschung in Deutschland $(\mathrm{N}=6)$.

\begin{tabular}{|c|c|c|}
\hline & & $\mathbf{N}$ \\
\hline \multirow[t]{5}{*}{ Bundesländer } & Nordrhein-Westfalen $[2,6]$ & 2 \\
\hline & Baden-Württemberg [1] & 1 \\
\hline & Bayern [3] & 1 \\
\hline & Niedersachsen [4] & 1 \\
\hline & Schleswig-Holstein [5] & 1 \\
\hline \multirow[t]{2}{*}{ Hochschulart } & Universität $[1,2,5]$ & 3 \\
\hline & (Fach-) Hochschule $[3,4,6]$ & 3 \\
\hline \multirow{6}{*}{$\begin{array}{l}\text { Denomination } \\
\text { (Mehrfachnen- } \\
\text { nung) }\end{array}$} & $\begin{array}{l}\text { Versorgungsforschung }[1,3,4,6] \\
\text { (angewandt [3]) }\end{array}$ & 4 \\
\hline & Versorgungswissenschaft [2] (-en [5]) & 2 \\
\hline & Implementierungswissenschaft [1] & 1 \\
\hline & $\begin{array}{l}\text { Gesundheits- und Versorgungswissen- } \\
\text { schaften [5] }\end{array}$ & 1 \\
\hline & $\begin{array}{l}\text { Versorgungsforschung und Management } \\
\text { im Gesundheitswesen [6] }\end{array}$ & 1 \\
\hline & Versorgungsforschung und -gestaltung [4] & 1 \\
\hline $\begin{array}{l}\text { Studiengangs- } \\
\text { profil }\end{array}$ & Master of Science [1-6] & 6 \\
\hline \multirow{2}{*}{$\begin{array}{l}\text { Art des } \\
\text { Studiengangs } \\
\text { (Mehrfachnen- } \\
\text { nung) }\end{array}$} & Konsekutiver Masterstudiengang [1-6] & 6 \\
\hline & $\begin{array}{l}\text { Master-Verbundstudiengang } \\
\text { (drei Fakultäten angehörig) [2] }\end{array}$ & 1 \\
\hline \multirow{4}{*}{$\begin{array}{l}\text { Fakultätszugehö- } \\
\text { rigkeit } \\
\text { (Mehrfachnen- } \\
\text { nung) }\end{array}$} & Medizinische Fakultät $[1,2,5]$ & 3 \\
\hline & $\begin{array}{l}\text { Wirtschafts- und Sozialwissenschaftliche } \\
\text { Fakultät }[2,4]\end{array}$ & 2 \\
\hline & Humanwissenschaftliche Fakultät [2 ] & 1 \\
\hline & Fakultät für Gesundheit und Pflege [3] & 1 \\
\hline \multirow{2}{*}{$\begin{array}{l}\text { Erstakkreditie- } \\
\text { rung }\end{array}$} & $2015[1-3]$ & 3 \\
\hline & $2017-2020[4-6]$ & 3 \\
\hline \multirow{4}{*}{$\begin{array}{l}\text { Anzahl } \\
\text { Studienplätze }\end{array}$} & Keine Zulassungsbegrenzung $[5,6]$ & 2 \\
\hline & $30[2]$ & 1 \\
\hline & $25[3,4]$ & 2 \\
\hline & $20[1]$ & 1 \\
\hline \multirow[t]{2}{*}{ Studienform } & Vollzeit $[1,2,4,5]$ & 4 \\
\hline & Teilzeit $[3,6]$ & 2 \\
\hline \multirow[t]{2}{*}{ Semesterstart } & Wintersemester $[1,2,4-6]$ & 5 \\
\hline & Sommersemester [3] & 1 \\
\hline \multirow[t]{2}{*}{ Anzahl Semester } & $5[6]$ & 1 \\
\hline & $4[1-5]$ & 5 \\
\hline \multirow{2}{*}{$\begin{array}{l}\text { Dauer des } \\
\text { Studiums (Jahre) }\end{array}$} & $2,5[6]$ & 1 \\
\hline & $2[1-5]$ & 5 \\
\hline \multirow[t]{2}{*}{ Anzahl ECTS } & $120[1,2,4-6]$ & 5 \\
\hline & $90[3]$ & 1 \\
\hline
\end{tabular}

[1] Ruprecht-Karls-Universität Heidelberg; [2] Universität zu Köln; [3] Katholische Stiftungshochschule München; [4] Hochschule Osnabrück; [5] Universität zu Lübeck; [6] Fliedner Fachhochschule Düsseldorf

tionsebene entwickelte Kategoriengerüst angewendet, um deduktive Zuordnungen vorzunehmen und um die induktive Kategorienbildung auf Subebene zu strukturieren. Redundanzen wurden entfernt und die Abstraktionsebenen des Datenmaterials angeglichen und kohärent dargestellt.

\section{Ergebnisse}

Die Abstraktionsebenen der analysierten Inhalte der Modulhandbücher variierten zwischen 2 oder 4 Ebenen. Hierbei wurden 89 Module mit den jeweiligen Bezeichnungen (ohne Anwendungsmodule (außer Module mit Projektbezeichnung), z. B. Praktika), 114 Subkategorien und 579 Sub-subkategorien mit Lehr-Lerninhalten und punktuell weitere dazugehörige Beschreibungen analysiert.

Die analysierten Studiengänge der Versorgungsforschung in Deutschland, mit einer Erstakkreditierung zwischen 2015 und 2020, schließen alle mit einem Master of Science ab. Bis auf eine Ausnahme mit einer 90 ECTS-Belegung sind sie mit 120 zu erreichenden ECTS ausgerichtet ( $\vee$ Tab. 1). Die Studiendauer beträgt bei den Studiengängen in Vollzeit zwei Jahre, in den Teilzeitstudiengängen, abhängig von der Anzahl ECTS, zwei bis zweieinhalb Jahre. Die Denominationen der Studiengänge umfassen die Versorgungsforschung, -gestaltung und -wissenschaft mit zusätzlichen Bezeichnungen wie Implementierungswissenschaft, Gesundheitswissenschaften und Management. Zwei Studiengänge werden in Nordrhein-Westfalen, jeweils 1 Studiengang wird in Baden-Württemberg, Bayern, Niedersachsen und Schleswig-Holstein angeboten. Die Hälfte aller Studiengänge ist an Universitäten angesiedelt.

Die Zulassungsvoraussetzungen umfassen ein vorheriges Studium, Bachelor- oder Diplomstudium bspw. von wenigstens 6 Semestern oder 180 ECTS oder mehr. In einem Studiengang ist eine Berufszulassung in einem Gesundheitsberuf notwendig. Die zulassungsrelevanten Bezugswissenschaften sind die Gesundheits-, Therapie-, Pflege-, Hebammenwissenschaft und Sozialwissenschaften. An den Hochschulen sind weitere Kenntnisse im Bereich der empirischen Sozialforschung (min. 6 ECTS) und/oder eine Abschlussnote von 2,5 oder 2,7 und besser für die Zulassung von Bedeutung. Ebenso können Fachkenntnisse in ausgewiesenen Bereichen sowie Sprachkenntnisse zulassungsrelevant sein ( $\triangleright$ Tab. 2 ).

Die jeweiligen Studiengänge haben zum Ziel, Studierende durch die Aneignung von Fachwissen, Forschungs- und Methodenkompetenzen sowie fachübergreifenden, personalen und sozialen Kompetenzen zu befähigen, die Studienziele zu erreichen. Managementkompetenzen und ein Verständnis von Rollen in der (Versorgungs-) Forschung z. B. der Bezugsdisziplinen, der Leistungserbringer und der Patienten, sind hierbei ebenso von Bedeutung. Die Studierenden sollen in die Lage versetzt werden, Versorgungsbedarfe zu identifizieren, Innovationen der Versorgung im Gesundheitswesen zu entwickeln, zu initiieren und zu evaluieren. Eine kritische Auseinandersetzung mit den Veränderungen im Gesundheitswesen, der Gestaltung und der Verbesserung der Qualität im Versorgungssystem soll gefördert sowie Voraussetzungen geschaffen werden, Innovationen in der Gesundheitsversorgung methodisch zu planen, durchzuführen, zu analysieren und zu implementieren. Es geht hierbei z. B. um adressatInnengerechte Versorgungsangebote, -strukturen und -prozesse, patientenzentrierte Gesundheitsversorgung, Patientensicherheit, Nachhaltigkeit und soziale Gerechtigkeit in der Versorgung sowie die Vermeidung von Unter-, Über- oder Fehlversorgung. 
- Tab. 2 Zulassungsvoraussetzungen Studiengänge der Versorgungsforschung in Deutschland $(\mathrm{N}=6)$.

\begin{tabular}{|c|c|c|c|}
\hline & & & $\mathbf{N}$ \\
\hline \multirow{17}{*}{$\begin{array}{l}\text { Zulassungs- } \\
\text { voraussetzungen } \\
\text { (Mehrfachnennung) }\end{array}$} & \multirow{4}{*}{$\begin{array}{l}\text { Vorheriges } \\
\text { Studium }\end{array}$} & Bachelorstudium [1-6] & 6 \\
\hline & & Oder Berufsqualifizierender Abschluss (180 ECTS oder >) [6] & 1 \\
\hline & & $\begin{array}{l}\text { Bachelor- oder Diplomstudiengangs, im Umfang von wenigstens } 6 \text { Semestern (als } \\
\text { Vollzeitäquivalent) [3] }\end{array}$ & 1 \\
\hline & & $\begin{array}{l}\text { An einer deutschen Hochschule oder ein gleichwertiger Abschluss an einer ausländischen } \\
\text { Hochschule }[3,6]\end{array}$ & 2 \\
\hline & \multirow{6}{*}{$\begin{array}{l}\text { Bezugswissen- } \\
\text { schaften sind } \\
\text { bspw. }\end{array}$} & Gesundheitswissenschaften $[1-3,5,6]$ & 5 \\
\hline & & Therapiewissenschaft [1] & 1 \\
\hline & & Pflegewissenschaft $[1,3,6]$ & 3 \\
\hline & & Hebammenwissenschaft [1] & 1 \\
\hline & & Sozialwissenschaften $[1,6]$ & 2 \\
\hline & & Medizin [2] & 1 \\
\hline & Berufszulassung & Gesundheitsberufe (Hebammenwesen, Ergotherapie, Logopädie, Physiotherapie oder Pflege) [4] & 1 \\
\hline & \multirow{4}{*}{$\begin{array}{l}\text { Weitere } \\
\text { Kenntnisse }\end{array}$} & Methodenkenntnisse empirischer Sozialforschung $[1,2,5]$ (min. 6 ECTS) [1] & 3 \\
\hline & & $\begin{array}{l}\text { Fachkenntnisse evidenzbasierter Praxis/Gesundheitsversorgung, zu medizinischen Grundlagen } \\
\text { sowie im Bereich Gesundheitssystem/ Gesundheitsökonomie bzw. Qualitätsmanagement [5,6] }\end{array}$ & 2 \\
\hline & & Gesundheits- und Sozialwesen [2] & 1 \\
\hline & & Sprachkenntnisse (Englisch, Deutsch) $[1,4]$ & 2 \\
\hline & \multirow[t]{2}{*}{ Abschlussnote } & 2,5 oder besser $[3,6]$ & 2 \\
\hline & & 2,7 oder besser [5] & 1 \\
\hline
\end{tabular}

[1] Ruprecht-Karls-Universität Heidelberg; [2] Universität zu Köln; [3] Katholische Stiftungshochschule München; [4] Hochschule Osnabrück;

[5] Universität zu Lübeck; [6] Fliedner Fachhochschule Düsseldorf

\section{Lehr-Lerninhalte der Studiengänge der Versorgungsforschung}

Insgesamt bilden 5 Inhaltscluster die Lehr-Lerninhalte ab: (1) Wissenschaften im Kontext der Versorgungsforschung, (2) Grundlagen und Anwendungsfelder der Versorgungsforschung, (3) Methodik und wissenschaftliches Arbeiten (4) Versorgung im Gesundheitswesen und (5) Ethik in Forschung und Praxis. Es zeigt sich, dass bis auf wenige studiengangsspezifische Schwerpunkte die LehrLerninhalte über die Studiengänge hinweg ähnlich strukturiert sind. Folgende Lehr- Lerninhalte sind nahezu in allen Studiengängen zu finden ( $\triangleright$ Tab. 3 und 4): Versorgungswissenschaft/Versorgungsforschung, Implementierungswissenschaft/ -forschung, Epidemiologie, Gesundheitsökonomie, Methodik und wissenschaftliches Arbeiten, Management im Gesundheitswesen und Ethik. Als besondere Schwerpunkte der Studiengänge der Versorgungsforschung sind an der Ruprecht-Karls-Universität Heidelberg die Implementierungswissenschaft, an der Universität zu Köln die organisationsbezogene Versorgungswissenschaft zu sehen. Hierbei beinhaltet die Implementierungswissenschaft “(...) die wissenschaftliche Studie von Methoden, um die systematische Aufnahme Forschungsergebnissen und anderen evidenzbasierten Praxen in den Routinealltag mit dem Ziel die Qualität und Wirksamkeit der Versorgung zu verbessern“ (Übersetzung SK) [14]. Die organisationsbezogenen Versorgungsforschung untersucht Organisationsstrukturen und -prozesse von Versorgungsorganisationen und ihre Determinanten und Folgen und strebt auf dieser Basis „(...) neben dem Erkennen systematischer Einflüsse auf Versorgungsoutcomes - insbesondere die Verbesserung und Neuentwicklung von Maßnahmen zur Steigerung der Versorgungsqualität an“ [2]. An der Katholische Stiftungshochschule München sind die Anthropologie, die Ethik und die altersdifferenzierte Versorgung im Kontext der Pflegewissenschaft und Gesundheitswissenschaften und an der Hochschule Osnabrück sind die berufsgruppenspezifische Versorgungsforschung (Pflege,- Therapie- und Hebammenwissenschaft) im Hinblick auf die Versorgungsqualität und Evaluation gesundheitlicher Dienstleistungen ausgewiesene Lehr-Lernschwerpunkte. An der Universität zu Lübeck sind die Gesundheitswissenschaft aus Sicht der Sozialwissenschaften, Evaluationsforschung und die Versorgungstechnologien (Digitales Gesundheitswesen) sowie an der Fliedner Fachhochschule Düsseldorf die Ausrichtung auf die Qualitäts- und Patientensicherheitsforschung sowie die Implementierung von Versorgungsinnovationen als besondere Studienschwerpunkte zu sehen.

\section{Wissenschaften im Kontext der Versorgungsforschung}

Die Lehr- Lerninhalte der jeweiligen Wissenschaften umfassen überwiegend die Nomenklatur, einschließlich Klassifikationen, die Theorien, die Modelle, die Rahmenkonzepte und die Ansätze, die durch die Wissenschaftsströmungen geprägt sind. Ebenso ist die Theorie- und Methodenentwicklung beschrieben. Darüber hinaus werden in der Versorgungswissenschaft und den Gesundheitswissenschaften inhaltliche Schwerpunkte, z. B. der Medizin, gesetzt. Als Themenschwerpunkte werden Prävention, Gesundheitsförderung und soziale Gerechtigkeit beschrieben. Die Lehr-Lerninhalte der Implementierungswissenschaft umfassen die Implementierungsforschung, die Wissenstranslation, die Dissemination und Evaluation und schließen entsprechend empirische Befunde mit ein. Im Rahmen der Epidemiologie werden klinische, umweltbezogene und geografische Aspekte abgebildet. Auch bevölkerungsbezogene Kennzahlen und die Gesundheitsberichterstattung sind von Bedeutung. Im Rahmen der Pflege- 
> Tab. 3 Lehr-Lerninhalte der Studiengänge der Versorgungsforschung $(N=6)$.

\begin{tabular}{|c|c|c|}
\hline Hauptkategorien & Subkategorien & Sub-Subkategorien \\
\hline \multicolumn{3}{|c|}{ 1) WISSENSCHAFTEN IM KONTEXT DER VERSORGUNGSFORSCHUNG } \\
\hline \multirow[t]{2}{*}{$\begin{array}{l}\text { Versorgungswissenschaft } \\
{[2,3,5,6]}\end{array}$} & & $\begin{array}{l}\text { Theorien, Modelle, Rahmenkonzepte und Ansätze * [1,2,6] (aus Sicht der } \\
\text { Sozialmedizin, Medizinsoziologie, Medizinpsychologie, Gesundheitsökonomie [2]) }\end{array}$ \\
\hline & & Informatik für Gesundheits- und Versorgungswissenschaften ${ }^{*}[5]$ \\
\hline \multirow[t]{5}{*}{ Gesundheitswissenschaften * } & & $\begin{array}{l}\text { Theorien, Modelle von Gesundheit und Krankheit* }[2,5] \text { (aus Sicht der Sozialwis- } \\
\text { senschaften [5]) }\end{array}$ \\
\hline & & Theorie- und Methodenentwicklung (national und international) $*[3,5]$ \\
\hline & & Nomenklatur * und Konzepte der Gesundheitsversorgung * $[2,5]$ \\
\hline & & $\begin{array}{l}\text { Gesundheitsförderung und Prävention * }[2,5] \text { (Determinanten von Gesundheit und } \\
\text { Krankheit }{ }^{*}[2,5] \text { Gesundheitskompetenz }{ }^{*}[2,5]\end{array}$ \\
\hline & & Soziale Gerechtigkeit* [5]) \\
\hline \multirow[t]{2}{*}{ Medizinsoziologie [2] } & & Theorien, Rahmenmodelle [2] \\
\hline & & Medizinsoziologische Methoden [2] \\
\hline \multirow{2}{*}{$\begin{array}{l}\text { Arbeits-, Sozial- und klinische } \\
\text { Umweltmedizin [2] }\end{array}$} & & Wissenschaftliche Grundlagen [2] \\
\hline & & $\begin{array}{l}\text { Prävention und Gesundheitsförderung in der Arbeitsmedizin * [2] (Determinanten } \\
\text { von Gesundheit und Krankheit* [2]) }\end{array}$ \\
\hline \multirow[t]{9}{*}{$\begin{array}{l}\text { Improvement- / Implemen- } \\
\text { tierungswissenschaft }[1,3,6]\end{array}$} & \multirow[t]{2}{*}{$\begin{array}{l}\text { Implementierungsforschung } \\
{[4,6]}\end{array}$} & $\begin{array}{l}\text { Theorien, Modelle, Rahmenkonzepte und Ansätze * }[1-4,6] \text { (aus Sicht z. B. der } \\
\text { Sozialpsychologie, Soziologie [2], im Kontext der Pflege- und Gesundheitswissen- } \\
\text { schaft [3]) }\end{array}$ \\
\hline & & Gegenstände* $[5]$ \\
\hline & $\begin{array}{l}\text { Interventionen der } \\
\text { Implementierung }{ }^{*}[1,2]\end{array}$ & \\
\hline & \multirow{2}{*}{$\begin{array}{l}\text { Prozesse der Implementie- } \\
\text { rung }{ }^{*}[1,4]\end{array}$} & Einflussfaktoren * $[1,4]$ \\
\hline & & Gestaltung von Implementierungsprozessen $[1,4]$ \\
\hline & $\begin{array}{l}\text { Wissenstranslation }[2-4,6] \\
\text { und -transfer [1] }\end{array}$ & \\
\hline & Dissemination $[3,6]$ & Theorien, Modelle, Rahmenkonzepte und Ansätze * $[3,6]$ \\
\hline & $\begin{array}{l}\text { Evaluation (formativ, } \\
\text { summativ) }[2,5]\end{array}$ & Evaluationsforschung [2,5], Methodologie [5] \\
\hline & Evidenzbasierung $[1,2]$ & \\
\hline \multirow{2}{*}{$\begin{array}{l}\text { Organisationswissenschaften } \\
{[2,6](-e n[2])}\end{array}$} & \multirow{2}{*}{$\begin{array}{l}\text { Organisationsforschung und } \\
\text { Organisationsdiagnostik [2] }\end{array}$} & Empirische Methoden * $[2,6]$ \\
\hline & & $\begin{array}{l}\text { Theorien, Modelle, Rahmenkonzepte und Ansätze * [2,6] (aus Sicht der Soziologie, } \\
\text { Psychologie und ökonomischen Organisationstheorie [2]) }\end{array}$ \\
\hline \multirow[t]{7}{*}{ Epidemiologie $[1,2,4,5]$} & \multirow[t]{5}{*}{ Grundlagen* } & Nomenklatur ${ }^{*}[1,2]$ \\
\hline & & Gegenstände * $[1,4]$ \\
\hline & & Kennzahlen * Public Health $[1,4]$ \\
\hline & & Gesundheitsberichterstattung [1] \\
\hline & & Forschungsdesigns * $[1,2,4,5]$ Versorgungsepidemiologische Methoden $[6]$ \\
\hline & Klinische Epidemiologie $[2,5]$ & Epidemiologische Studien der Umweltmedizin [2] \\
\hline & $\begin{array}{l}\text { Geografische Epidemiologie } \\
\text { [5] }\end{array}$ & \\
\hline $\begin{array}{l}\text { Pflegewissenschaft [3,4]/ } \\
\text { Therapiewissenschaft [4,5]/ } \\
\text { Hebammenwissenschaft [4] }\end{array}$ & $\begin{array}{l}\text { Projekt Forschung in den } \\
\text { Therapieberufen [5] }\end{array}$ & \\
\hline \multirow[t]{4}{*}{ Gerontologie [2] } & Gerontopsychiatrie [2] & Theorie- und Methodenentwicklung (national und international) * [3] \\
\hline & $\begin{array}{l}\text { Soziologie, Demografie, } \\
\text { Ökonomie, Psychologie, } \\
\text { Ethik [2] }\end{array}$ & \\
\hline & Recht $[2,3]$ & \\
\hline & Gerontologische Praxis [2] & Altersdifferenzierte Versorgungsbedarfe und -konzepte [3] \\
\hline \multirow{4}{*}{$\begin{array}{l}\text { Gesundheitsökonomie } \\
{[1,2,6]}\end{array}$} & \multirow{4}{*}{$\begin{array}{l}\text { Gesundheitsökonomische } \\
\text { Evaluation }[1,2]\end{array}$} & Politische Gegenstände * $[1,3]$ \\
\hline & & Krankheitsbezogene und Patientenbezogene Gegenstände * [2] \\
\hline & & 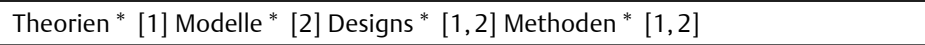 \\
\hline & & Versorgungsformen ${ }^{*}[2]$ \\
\hline
\end{tabular}


Tab. 3 Fortsetzung

\begin{tabular}{|c|c|c|}
\hline Hauptkategorien & Subkategorien & Sub-Subkategorien \\
\hline & & Kosten Nutzen * [1,6] \\
\hline & & Gesundheitsökonomische Auswirkungen und Erfordernisse [5] \\
\hline \multirow[t]{3}{*}{ Anthropologie [3] } & $\begin{array}{l}\text { Philosophische Anthropolo- } \\
\text { gie [3] }\end{array}$ & \\
\hline & $\begin{array}{l}\text { Kultur- und Sozialanthropo- } \\
\text { logie [3] }\end{array}$ & \\
\hline & Leibphänomenologie [3] & \\
\hline \multicolumn{3}{|c|}{ 2) GRUNDLAGEN UND ANWENDUNGSFELDER DER VERSORGUNGSFORSCHUNG } \\
\hline \multirow[t]{12}{*}{ Versorgungsforschung [1-6] } & \multirow{4}{*}{$\begin{array}{l}\text { Grundlagen der Versorgungs- } \\
\text { forschung * }\end{array}$} & Nomenklatur * [4] \\
\hline & & Verortung * (national, international) [3-4] \\
\hline & & $\begin{array}{l}\text { Theorien, Modelle, Rahmenkonzepte und Ansätze * [1-4] Theorien und Modelle der } \\
\text { personenzentrierten Versorgung [5] }\end{array}$ \\
\hline & & Politische Steuerung * [3] \\
\hline & $\begin{array}{l}\text { Interdisziplinäre Versor- } \\
\text { gungsforschung [4] }\end{array}$ & \\
\hline & $\begin{array}{l}\text { Berufsgruppenspezifische } \\
\text { Versorgungsforschung [4] }\end{array}$ & Heilberufe * wie Pflege- undTherapieberufe * [4] \\
\hline & $\begin{array}{l}\text { Berufsgruppenspezifische } \\
\text { Vertiefung/ Fallarbeit (H, E, L, } \\
\text { P, P) [4] }\end{array}$ & Fall- und Patientenorientierung [4] \\
\hline & \multirow{5}{*}{$\begin{array}{l}\text { Qualitäts- und Patientensi- } \\
\text { cherheitsforschung [6] }\end{array}$} & Theorien, Modelle, Rahmenkonzepte und Ansätze * [5,6] Methoden [2] \\
\hline & & Gegenstände * [2, 5, 6]: Innovationen [6] Patientenpartizipation * [1,5] \\
\hline & & Einflussfaktoren * [5] \\
\hline & & Interventionen * , Entwicklung und Evaluation komplexer Interventionen [5] \\
\hline & & Messung * $[5]$ \\
\hline \multirow{4}{*}{$\begin{array}{l}\text { Fachbezogene Versorgungs- } \\
\text { forschung * }\end{array}$} & \multirow[t]{2}{*}{ Medizin [2] } & Relevante Krankheitsbilder und Kasuistik I und II [2] \\
\hline & & Medizinische Fachsprache und Kasuistik I [2] \\
\hline & $\begin{array}{l}\text { Allgemeine Heilpädagogik } \\
\text { [2] }\end{array}$ & Normative Probleme und Ethik [2] \\
\hline & Rehabilitation [5] & Lernprinzipien [5] \\
\hline \multirow{11}{*}{$\begin{array}{l}\text { Anwendungsfelder der } \\
\text { Versorgungsforschung } \\
{[1,3,4]}\end{array}$} & & Versorgungsqualität* $[4,5]$ \\
\hline & & Sektorübergreifende Versorgungskonzepte * $[6]$ \\
\hline & & Patientenorientierung $[1,6]$ \\
\hline & & Regionaldifferenzierte Versorgungsbedarfe und-konzepte $[2,3]$ \\
\hline & & Altersdifferenzierte Versorgungsbedarfe und -konzepte [3] \\
\hline & & Evaluation gesundheitlicher Dienstleistungen [4] \\
\hline & & Familie und Lebenswelt [4] \\
\hline & & Versorgung im internationalen Kontext [4] \\
\hline & & Professionsentwicklung und interdisziplinäre Versorgungsgestaltung [4] \\
\hline & & $\begin{array}{l}\text { Technische Innovationen, E-Health und digitales Gesundheitswesen [3-6] } \\
\text { Technische Unterstützung von Versorgungsprozessen [4] Technologien in der } \\
\text { Gesundheitsversorgung [5] Mensch-Maschine-Systeme [5] }\end{array}$ \\
\hline & & Ausgewählte * Anwendungsfelder $[1,2]$ \\
\hline \multicolumn{3}{|c|}{ 3) METHODIK UND WISSENSCHAFTLICHES ARBEITEN } \\
\hline \multirow[t]{2}{*}{ Wissenschaftstheorie * $[3,6]$} & \multirow[t]{2}{*}{ Erkenntnistheorien [3] } & Allgemeine Erkenntnislehre $[3,6]$ \\
\hline & & Anthropologie [3] und Philosophie * [3] \\
\hline $\begin{array}{l}\text { Wissenschaftliche [1] } \\
\text { (Forschungs-) Designs [1-6] }\end{array}$ & $\begin{array}{l}\text { Forschungsrichtungen }{ }^{*} \\
{[1-3]}\end{array}$ & Entwicklung und Evaluation* $[3,5]$ \\
\hline
\end{tabular}


Tab. 3 Fortsetzung

\begin{tabular}{|c|c|c|}
\hline Hauptkategorien & Subkategorien & Sub-Subkategorien \\
\hline & $\begin{array}{l}\text { Grundlagen empirischer } \\
\text { Forschung [2] }\end{array}$ & $\begin{array}{l}\text { Designs und Methoden * [1] Forschungsmethoden in den Gesundheitswissenschaf- } \\
\text { ten [5] }\end{array}$ \\
\hline & \multirow[t]{3}{*}{ Quantitative Forschung [1-6] } & Forschungsdesigns * $[1,5]$ \\
\hline & & Statistische Verfahren und Maße * [1-6] \\
\hline & & Instrumentenentwicklung und -testung ${ }^{*}[3]$ \\
\hline & \multirow{3}{*}{$\begin{array}{l}\text { Qualitative Forschung } \\
{[1-3,5-6]}\end{array}$} & Designs und Methodologien ${ }^{*}[3,5,6]$ \\
\hline & & Methodik* [3] \\
\hline & & Evaluation [3] \\
\hline & $\begin{array}{l}\text { Mixed-Methods und } \\
\text { Triangulation }[2,3,6]\end{array}$ & \\
\hline & \multirow{6}{*}{$\begin{array}{l}\text { Besondere Methoden der } \\
\text { Datenerhebung \& -analyse in } \\
\text { der Versorgungsforschung } \\
{[2,3,6]}\end{array}$} & Modellierung [6] \\
\hline & & $\begin{array}{l}\text { Methoden der organisationsbezogenen Versorgungsforschung (z. B. Mehrebenen- } \\
\text { analysen) [6] }\end{array}$ \\
\hline & & Register als Evaluationsinstrumente [2] \\
\hline & & Patientenberichtete Zielgrößen * $[2,5,6]$ \\
\hline & & Big Data Analysen * [6] \\
\hline & & Routinedatenanalysen * [6] \\
\hline & \multirow[t]{3}{*}{ Studiengüte * $[3,5,6]$} & Gütekriterien * $[3,5,6]$ \\
\hline & & Kontrolle $^{*}[2]$ \\
\hline & & Wissenschaftliche Standards* $[5,6]$ \\
\hline & \multirow{3}{*}{$\begin{array}{l}\text { (Systematische-) Literaturar- } \\
\text { beiten * }[1-3,5,6]\end{array}$} & (Systematische-) Recherchen und Analysen * $[1-3,5,6]$ \\
\hline & & Evidenzsynthesen (qualitativ und quantitativ) $[2,5]$ \\
\hline & & Critical Appraisal * $[1,2,5]$ \\
\hline & \multirow{3}{*}{$\begin{array}{l}\text { Forschungsprozess [1, 3, 5] / } \\
\text { Theoretische Grundlagen [3] }\end{array}$} & Studienplanung (Protokolle) [1-5] \\
\hline & & Datenerhebung in der Versorgungsforschung $[1-3,5,6]$ \\
\hline & & Datenanalyse in der Versorgungsforschung $[1,2,6]$ \\
\hline \multirow[t]{8}{*}{ Wissenschaftsystem [1] } & Publizieren $[1,5]$ & $\begin{array}{l}\text { Veröffentlichung Studienergebnisse * Forschungsberichte }[1,3,5,6] \text { Peer Review } \\
\text { Methoden }[4,5]\end{array}$ \\
\hline & 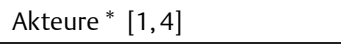 & \\
\hline & Forschungsförderung [1] & \\
\hline & Antragsstellung [3] & \\
\hline & $\begin{array}{l}\text { Studienprotokolle entwickeln } \\
\text { [5] }\end{array}$ & \\
\hline & \multirow{2}{*}{$\begin{array}{l}\text { Projektmanagement in } \\
\text { Forschung }[1,3,6] \text { und Praxis } \\
{[6]}\end{array}$} & Forschungsmanagement und -akquise [3] \\
\hline & & $\begin{array}{l}\text { Forschungsplanung und -umsetzung [4] Projektentwicklung, Projektdurchführung, } \\
\text { Projektabschluss und Evidence-based Management [6] Methoden und Instrumen- } \\
\text { te }{ }^{*}[6]\end{array}$ \\
\hline & $\begin{array}{l}\text { Wissenschaftliches Schreiben } \\
{[1,2] \text { Einsatz Software* }}\end{array}$ & \\
\hline \multicolumn{3}{|c|}{ 4) VERSORGUNG IM GESUNDHEITSWSEN } \\
\hline \multirow{5}{*}{$\begin{array}{l}\text { Gesundheitssystem [1] } \\
\text { (-Forschung [3]) }\end{array}$} & Gesundheitspolitik $[3,5,6]$ & \\
\hline & \multirow{4}{*}{$\begin{array}{l}\text { Versorgungsstrukturen und } \\
\text {-prozesse }[1-4,6]\end{array}$} & Akteure $*^{*}[1-3,6]$ \\
\hline & & Gestaltungsprinzipien des Gesundheitswesens [1,4] \\
\hline & & Systemfaktoren [2] \\
\hline & & Internationaler Vergleich $[1,3]$ \\
\hline \multirow[t]{2}{*}{ Versorgungssteuerung $[6]^{*}$} & & Anforderungen \& Versorgungsbedarfe im Gesundheitswesen $[2,3,6]$ \\
\hline & & Modelle, Methoden, Instrumente und Konzepte * $[2,6]$ \\
\hline
\end{tabular}


> Tab. 3 Fortsetzung

\begin{tabular}{|c|c|c|}
\hline Hauptkategorien & Subkategorien & Sub-Subkategorien \\
\hline \multirow[t]{14}{*}{$\begin{array}{l}\text { Management [1-3] im } \\
\text { Gesundheitswesen [2] }\end{array}$} & $\begin{array}{l}\text { Integriertes Management, } \\
\text { Changemanagement \& } \\
\text { Innovationen im Gesund- } \\
\text { heitswesen [6] }\end{array}$ & Theorien, Modelle, Methoden, Instrumente, Rahmenkonzepte und Ansätze * $[1,6]$ \\
\hline & \multirow{4}{*}{$\begin{array}{l}\text { Qualitäts- / Risikomanage- } \\
\text { ment im Gesundheitswesen } \\
{[1,2,4-6]}\end{array}$} & Theorien, Modelle, Methoden, Rahmenkonzepte und Ansätze * [1,2,4-6] \\
\hline & & Instrumente ${ }^{*}[1,2,4-6]$ \\
\hline & & Managementsysteme * [6] \\
\hline & & Berichterstattung * $[1,4]$ \\
\hline & Betriebswirtschaftslehre [1] & Betriebswirtschaft * $[2,6]$ \\
\hline & \multirow{6}{*}{$\begin{array}{l}\text { Recht }[1,6] \text { und Mediation } \\
{[1]}\end{array}$} & Sozialrecht * $[1,4,6]$ \\
\hline & & Arbeitsrecht* [1] \\
\hline & & Medizinrecht* ${ }^{[6]}$ \\
\hline & & Haftungsrecht* ${ }^{[1,6]}$ \\
\hline & & Recht $[1,3]$ in der Gesundheitsversorgung [4] \\
\hline & & Mediation * [1] \\
\hline & \multirow[t]{2}{*}{ Medizinisches Controlling [6] } & Compliance Management * $[6]$ \\
\hline & & Controlling * [6] \\
\hline \multirow{5}{*}{$\begin{array}{l}\text { Organisationsentwicklung } \\
{[1,2] \text { und Personalentwick- }} \\
\text { lung }[2]\end{array}$} & & Theorien, Rahmenkonzepte und Ansätze * [1,2] \\
\hline & & Organisationsentwicklung und organisationales Lernen $[1,5]$ \\
\hline & & $\begin{array}{l}\text { Organisationsberatung und angewandte Organisationsentwicklung [1] Theorien, } \\
\text { Methoden, Rahmenkonzepte und Ansätze * }{ }^{[1]}\end{array}$ \\
\hline & & Besondere Gegenstände der Personal- und Organisationsentwicklung * $[1,2,6]$ \\
\hline & & Evidenzbasierte Organisationsentwicklung * [2] \\
\hline \multicolumn{3}{|c|}{ 5) ETHIK IN FORSCHUNG UND PRAXIS } \\
\hline \multirow[t]{14}{*}{ Ethik [1-6] } & \multirow[t]{6}{*}{ Versorgungsethik [3] } & Anthropologie und (Rechts-) Philosophie * [2-4] \\
\hline & & Recht und Ethik [3] \\
\hline & & Christliche Soziallehre [3] \\
\hline & & Gerechtigkeit* $[2,4]$ \\
\hline & & Ethisches Handeln * [4] \\
\hline & & Professionalisierung * [3] \\
\hline & \multirow{3}{*}{$\begin{array}{l}\text { Ethik in Bildung und } \\
\text { Bildungsmanagement [3] }\end{array}$} & Anthropologie und Philosophie * [3] \\
\hline & & Gegenstände * Theorien und Konzepte * [3] \\
\hline & & Standards * [3] \\
\hline & \multirow[t]{5}{*}{ Forschungsethik $[1,3,4,6]$} & Forschungsethische Prinzipien und Regularien ${ }^{*}[3,5,6]$ \\
\hline & & Rechtliche Grundlagen * $[3,6]$ \\
\hline & & Gegenstände * [1-4] \\
\hline & & Ethische Begutachtung * $[1,3,5]$ \\
\hline & & Beratung * [3] \\
\hline
\end{tabular}

[1] Ruprecht-Karls-Universität Heidelberg; [2] Universität zu Köln; [3] Katholische Stiftungshochschule München; [4] Hochschule Osnabrück;

[5] Universität zu Lübeck; [6] Fliedner Fachhochschule Düsseldorf * durch den Autor entwickelte Kategorien

wissenschaft sind aktuelle Trends und die Professionalisierung von Bedeutung. Die Pflege-, Therapie- und Hebammenwissenschaft werden herangezogen, um inhaltliche und methodische Beispiele zu nutzen. Die Gerontologie umfasst Bedarfe, z. B. altersdifferenzierte Versorgungsbedarfe, die Bedarfsplanung, aber auch psychiatrische Grundlagen sowie soziologische, demografische, ökonomische, psychologische Fragestellungen sowie rechtliche und ethische Fragen.

\section{Grundlagen und Anwendungsfelder der Versorgungsforschung}

Die nationale und internationale Verortung der Versorgungsforschung und deren Grundlagen sind Lehr-Lerninhalt. Differenziert wird zwischen nutzerorientierter und partizipativer Versorgungsforschung, interdisziplinären Forschungsfeldern wie Pflege und Therapieberufe und der Schnittstellenforschung. Im Rahmen einer berufsspezifischen Vertiefung wird eine kombinierte Fall- und Patientenorientierung fokussiert. Die Qualitäts- und Patientensicherheitsforschung, die Einflussfaktoren auf die Patientensicherheit wie 


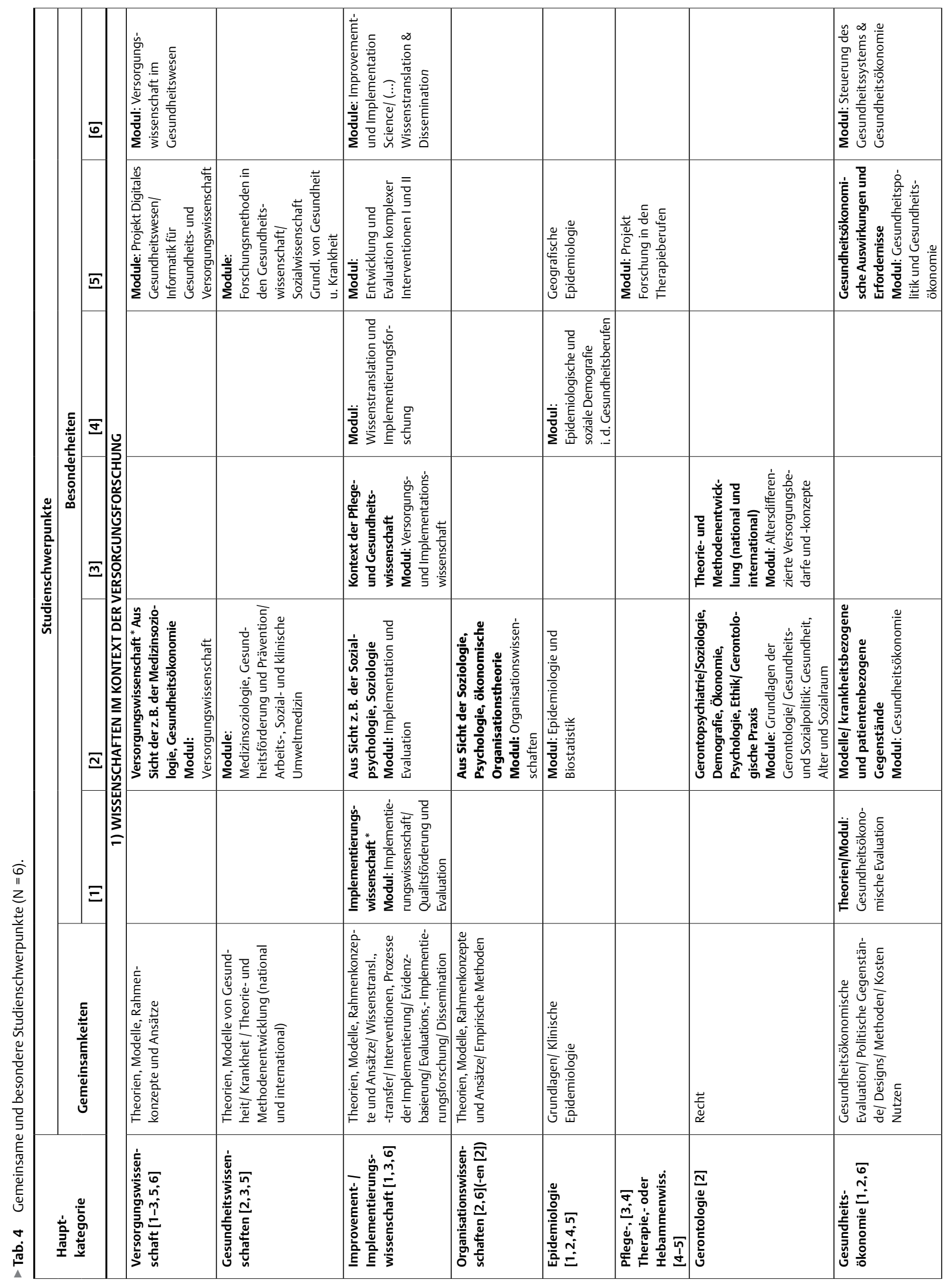




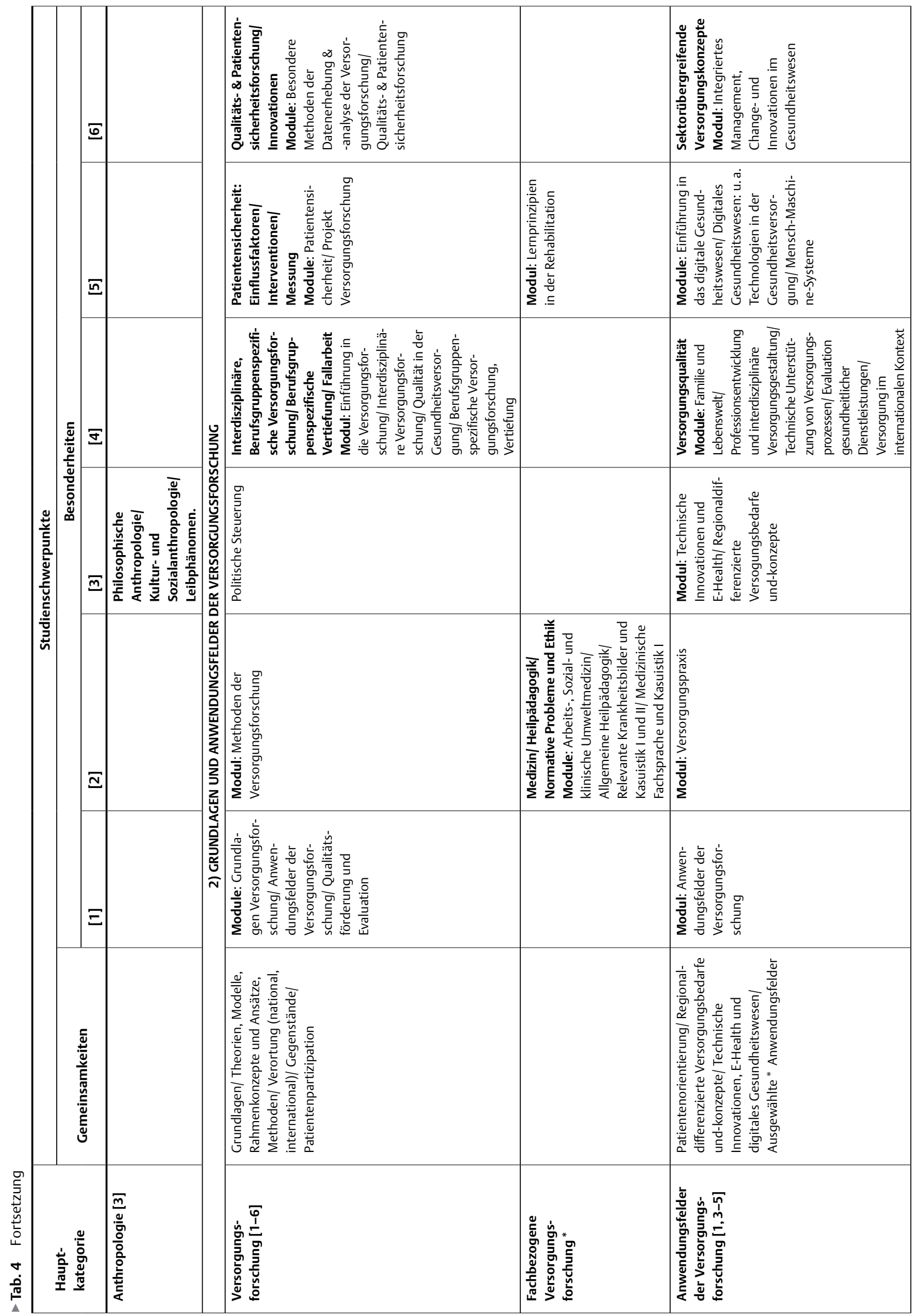




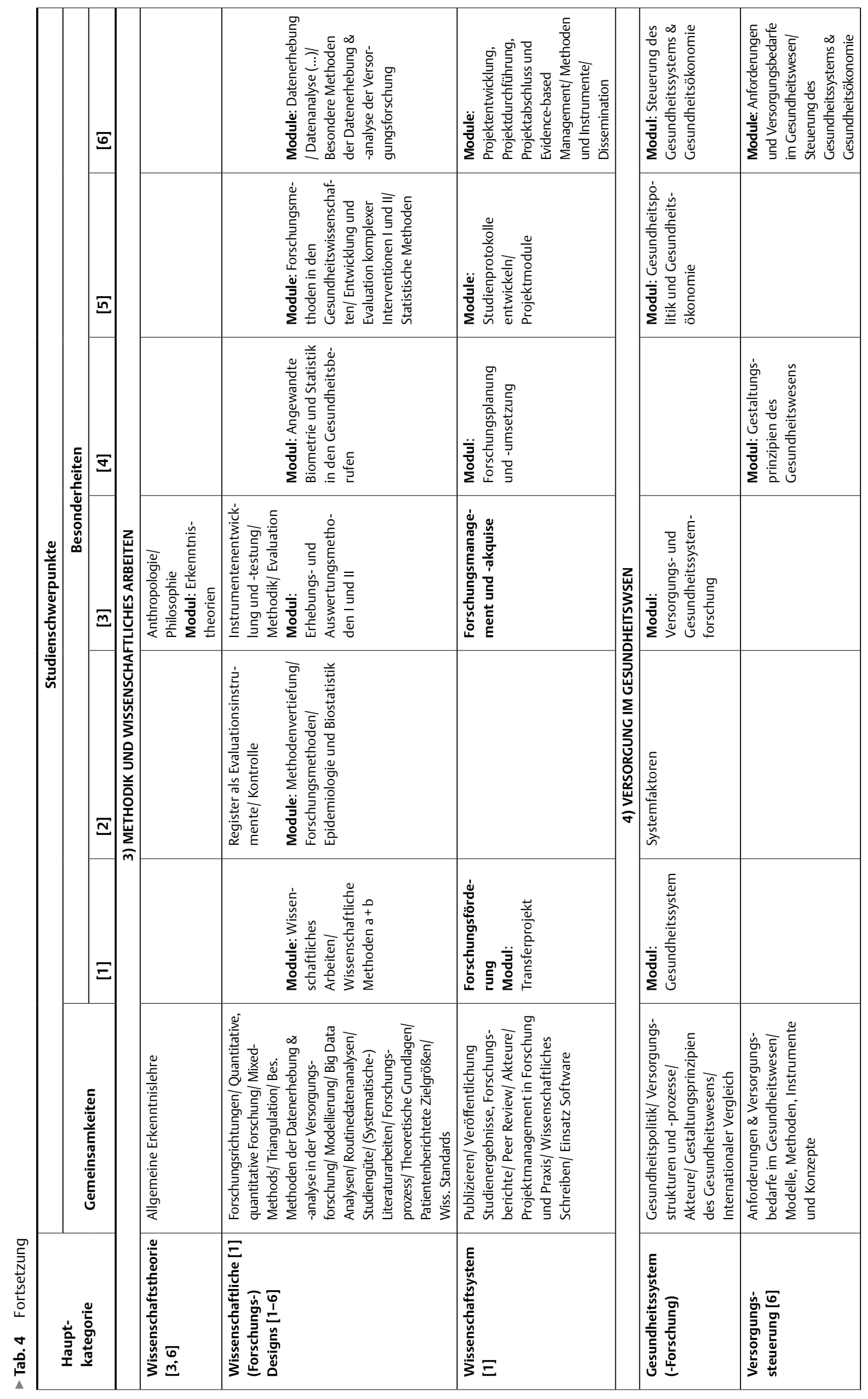




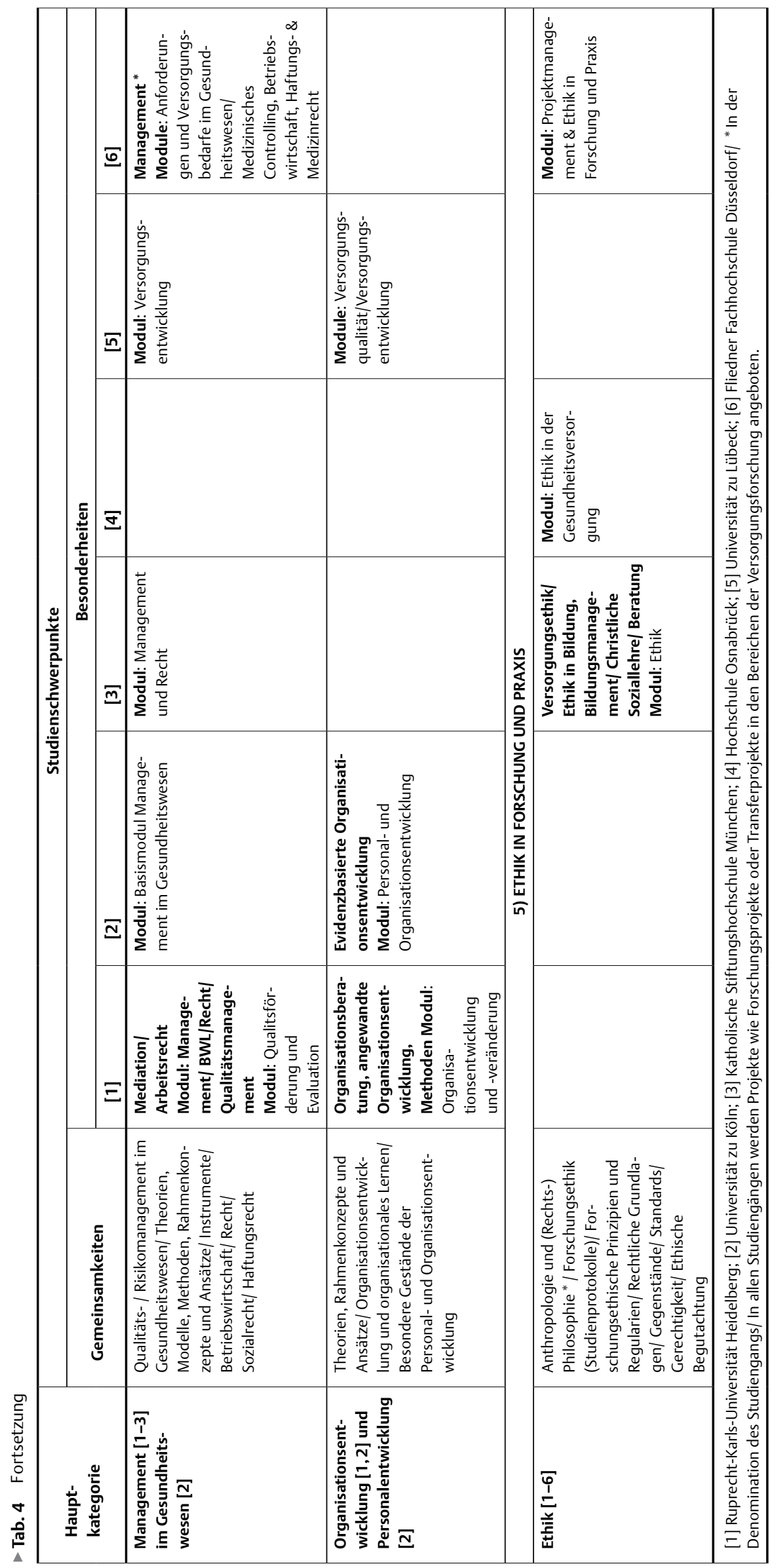


Sicherheitskultur, Kommunikation, Interventionen zur Implementierung, das Monitoring und die Messung sind ein Thema. Ein besonderes Augenmerk richtet sich auf die fachbezogene Versorgungsforschung. Die Anwendungsfelder der Versorgungsforschung umfassen beispielsweise die Versorgungsqualität, sektorübergreifende Versorgungskonzepte, die Berücksichtigung regional- und altersdifferenzierter Versorgungsbedarfe und -konzepte, die Evaluation gesundheitlicher Dienstleistungen, die Professionsentwicklung und interdisziplinäre Versorgungsgestaltung und technische Innovationen und insbesondere digitale Innovationen („digitales Gesundheitswesen“) sowie weitere ausgewählte Felder wie klinische Studien zu Medizinprodukten und diagnostischen Methoden.

\section{Methodik und wissenschaftliches Arbeiten}

Neben der Wissenschaftstheorie erstrecken sich die Lehr-Lerninhalte zu den wissenschaftlichen Designs über die Grundlagen-, die Interventions-, Evaluations- und partizipativen Forschung. Neben den Grundlagen zur empirischen Forschung, einschließlich des Forschungsprozesses, ist die quantitative und qualitative Forschung unter Berücksichtigung von Designs und Methodologien, Auswertungsverfahren und -maße Gegenstand der Lehre. Mixed MethodDesigns und Methoden der Triangulation sind ebenso von Bedeutung. Darüber hinaus sind die Merkmale und Voraussetzungen der Studienqualität wichtige Gegenstände der methodenorientierten Lehre. Konkrete Inhalte sind z. B. Gütekriterien, wissenschaftliche Standards und Anforderungen an die Durchführung und Berichterstattung von (systematischen-) Literaturarbeiten. Das Wissenschaftssystem und Themen wie Wissenschaftsakteure, Forschungsförderung, Antragsstellung, Projektmanagement, Publikationsqualifikationen sind weitere Inhalte der Modulhandbücher.

\section{Versorgung im Gesundheitswesen}

Die Lehr-Lerninhalte umfassen die Makro-, Meso- und Mikroebene der Versorgung unter Berücksichtigung der gesundheitspolitischen Strukturen und Prozesse. Hierbei werden Anforderungen und Versorgungsbedarfe im Gesundheitswesen thematisiert und ein integriertes Versorgungsmanagement, Qualitäts- und Risikomanagement, Changemanagement sowie die Organisations- und Personalentwicklung werden berücksichtigt. Ebenso sind die Betriebswirtschaftslehre, Medizinisches Controlling sowie Recht und Mediation Gegenstand der Lehr-Lerninhalte.

\section{Ethik in Forschung und Praxis}

Ethik in Bezug auf die Versorgung, unter Berücksichtigung ethischen Handelns, Gerechtigkeit und Professionalisierung, die Bildung unter Berücksichtigung von Standards und die Forschung einschließlich Beratung und Standards sind ebenso von Bedeutung.

\section{Anwendung der Lehr-Lerninhalte der Studiengänge der Versorgungsforschung}

Insgesamt zeigt sich, dass verschiedene Methoden zur Förderung der Anwendung der Lehr-Lerninhalte in den Modulhandbüchern verankert sind. Diese umfassen Begleitseminare, um die erworbenen Kenntnisse und Kompetenzen anhand der Lektüre einschlägiger Studien und Planung beispielhafter Forschungsprojekte zu vertiefen, Forschungswerkstätten, um den Forschungsprozess zu durchlaufen und dessen methodische Herausforderungen kennenzulernen, Pro- jekte zu spezifischen Anwendungsfeldern der Versorgungsforschung sowie als Möglichkeit, Forschungsprojekte und deren Management, teils über drei Semester, zu erproben. Darüber hinaus bieten Forschungspraktika die Möglichkeit der Praxisvernetzung, feldbezogen Versorgung zu analysieren und Forschungsmethoden zu erproben. Masterarbeiten und Forschungskolloquien runden die Möglichkeit, Versorgungsforschung anzuwenden und zu reflektieren, ab.

\section{Diskussion}

Das Ziel war es, die derzeit in Deutschland akkreditierten Versorgungsforschungsstudiengänge zu beschreiben und diese im Hinblick auf ihre Strukturdaten und ihre Inhalte zu analysieren sowie darauf basierend eine Diskussionsgrundlage für ein konsentiertes Kerncurriculum zu schaffen. Die 6 analysierten Studiengänge zeigen sich hinsichtlich ihrer strukturellen Merkmale und lehrlerninhaltlichen Ausrichtung in den wesentlichen Punkten als homogen. Ziel aller Studiengänge ist es, fach- und disziplinübergreifende personale und soziale Kompetenzen zu fördern, um Versorgungsbedarfe im Gesundheitswesen zu identifizieren, Innovationen der Versorgung zu entwickeln, zu initiieren, zu evaluieren und kritisch zu reflektieren.

Die Lehr-Lerninhalte decken sich in Bezug auf Terminologie, Rahmenkonzepte, Modelle, Theorien, empirische Versorgungsforschungsmethoden sowie hinsichtlich der System-, Professions- und Institutionsfaktoren mit den derzeitig beschriebenen Gegenständen und den Praxisanforderungen. Dies zeigt eine Analyse der Stellenausschreibungen im Gebiet der Versorgungsforschung. Die häufigsten Stellen werden in den Bereichen Wissenschaft und Forschung, Interessensvertretung, Datenmanagement und Versorgungsmanagement ausgeschrieben. Zu den benannten Qualifikationsprofilen gehören insbesondere Methodenkompetenzen und Kenntnisse des deutschen Gesundheitswesens [15]. Es existieren mittlerweile zahlreiche Health Services Research Masterstudiengänge an US-amerikanischen (z. B. Ann Arbor, Boston, Chicago, New York), kanadischen (z. B. Toronto, Halifax), britischen (z. B. Newcastle, Rochester) und australischen (z. B. Sydney) Universitäten. Diese sind zumeist an den Schools of Public Health oder an den Medizinischen Fakultäten der jeweiligen Standorte angesiedelt. An der Stanford University sind Ausbildung und Forschung in den Bereichen Versorgungsforschung und Medizinische Informatik eng vernetzt [16]. Am Standort Lübeck wird diese Vernetzung von Versorgungsforschung und Informatik bereits praktiziert, insbesondere durch die Vertiefungsrichtung „Digitales Gesundheitswesen“ in dem Studiengang „Gesundheits- und Versorgungswissenschaften“. Ein solches Vorgehen könnte auch an deutschen Hochschulstandorten in Deutschland Synergien schaffen und auf die interdisziplinären Arbeitsanforderungen in der Versorgungsforschung vorbereiten.

Im Hinblick auf den Forschungsgegenstand können traditionell die Studiengänge der Versorgungsforschung, der Public Health und Gesundheitsökonomie voneinander unterschieden werden [2]. Dennoch können ebenso Überschneidungen aufgrund engen Beziehungen zu den Disziplinen wie auch Gegenstände, in denen die Versorgungsforschung über die Forschungsdisziplinen hinaus geht, sichtbar gemacht werden. Die interprofessionell geprägte Versorgungsforschung mit den Alltagsbedingungen der Versorgung im Fokus geht 
über die individuellen Disziplinen Public Health und Gesundheitsökonomie (und weitere) hinaus. Sie hat zum Ziel „(...) ausgehend von der Patienten- sowie Populationsperspektive und vor dem Hintergrund komplexer Kontextbedingungen die Versorgungsstrukturen und -prozesse der Gesundheitsversorgung [zu untersuchen], den Outcome auf der Ebene der Alltagsversorgung [zu beschreiben] und komplexe Interventionen zur Verbesserung der Versorgung zu [evaluieren] “ [2]. Public Health richtet sich auf die Bevölkerungspopulationen oder größere Subpopulationen vor dem Hintergrund von Krankheitsprävention und Gesundheitsförderung aus und berücksichtigt hierbei die Angemessenheit von Ressourcen unter ethischen, kulturellen und medizinischen Gesichtspunkten[17]. Die Gesundheitsökonomie richtet sich vorrangig auf die „Ressourcenallokation“ und die „Effizienz des Ressourceneinsatzes “ [2]. Traditionell steht Public Health für den gesundheitsbezogenen Populationsbezug, der in der Versorgungsforschung dann an Bedeutung gewinnt, wenn ein individueller Versorgungsbezug unter Alltagsbedingungen gegeben ist, wie es bspw. bei Maßnahmen der Patientenedukation der Fall sein kann. Schnittstellen zur Gesundheitsökonomie entstehen dann, wenn beispielsweise die „Versorgungseffizienz“, „sozioökonomische Krankheitslast“ oder „Implementierungskosten“ eine Rolle spielen [2]. Das Erkenntnisinteresse der Versorgungsforschung ist damit umfassender und bezieht sich vor dem Hintergrund der Alltagswirksamkeit auf das komplexe Zusammenspiel von Strukturen, Prozessen und Ergebnissen der Versorgung. In der Analyse konnten daher auch traditionelle Module von Public Health, z. B. Epidemiologie, und der Gesundheitsökonomie in den bestehenden Studiengängen der Versorgungsforschung identifiziert werden. International zeigt sich, dass die „Public Health-Forschung“, die „Krankenhausforschung“ und die „Pflegeforschung “ oft gemeinsam in den Dienst der „Community Health-Forschung“ gestellt werden [18].

Es wird im Bereich der Versorgungsforschung derzeit eher erwartet, dass die Masterstudierenden der Versorgungsforschung ihren Bachelorabschluss in einer wissenschaftlichen Bezugsdisziplin der Versorgungsforschung absolvieren, ggfs. kombiniert mit einem Gesundheitsberuf. Inwieweit dieses erforderlich ist, könnte ebenso zukünftig diskutiert werden. Verbleibstudien können Hinweise darauf geben, welche Qualifikationsniveaus im Zusammenhang mit den jeweiligen zukünftigen Arbeitsfeldern angemessen sind. Ebenso ließen sich auf entsprechender Datenbasis weitere Schwerpunkte für Lehr- Lerninhalte ableiten. Diese Ergebnisse könnten unter Berücksichtigung aktueller Diskurse und weiterer Erkenntnisse als Grundlage für Empfehlungen zur Entwicklung eines Kerncurriculums dienen. Beispiele für das Gelingen eines solchen Vorhabens sind das Kerncurriculum Pflegewissenschaft [19] oder das internationale Kerncurriculum Public Health [20-21].

\section{Limitationen}

Die Inhaltsanalyse stand aufgrund unterschiedlicher Ordnungslogiken der Modulhandbücher, Abstraktionsniveaus und Bezeichnungen teils ähnlicher Lehr-Lerninhalte vor besonderen Herausforderungen. Es kann angenommen werden, dass auch nicht explizierte Lehr -Lerninhalte möglicherweise im Rahmen der Veranstaltungen zum Tragen kommen. Schließlich könnte ein Bestätigungsfehler (Confirmation Bias) vorliegen. Die Analyse wurde durch einen Autor durchgeführt, der einem der zu analysierenden Studiengänge angehörig ist. Ein möglicher Bias wurde vermieden, indem alle füh- renden Angehörigen der Studiengänge die Möglichkeit wahrgenommen haben, das Analyseprotokoll kritisch zu kommentieren und die Analyse in Diskussion mit den Analyseverantwortlichen mitzugestalten. Zudem wurde eine der Koautorinnen (NE) supervidierend eingesetzt, die keinen der analysierten Studiengänge vertritt. Überprüfungen des Kodierungsprozesses und der Kodierqualität fanden in Zusammenarbeit mit einer weiteren Koautorin statt $(\mathrm{CH})$.

\section{FAZIT}

In Deutschland werden derzeit an 6 Universitäts- oder Fachhochschulstandorten Masterstudiengänge der Versorgungforschung angeboten. Die Inhalte der Studiengänge sind im Wesentlichen homogen und decken sich mit dem vorhandenen Wissen zu Gegenstand, Terminologie, Anwendungsfeldern und Methoden der Versorgungsforschung. Dennoch geben die Standorte ihrem jeweiligen Studienangebot eine spezifische Ausrichtung. Aufgrund der Überschneidungen zu verwandten Studiengängen wie beispielsweise Public Health ist korrespondierend zur weiteren Entwicklung des Forschungsbedarfs in diesen Disziplinen und im Rahmen der Qualitätssicherung der Studiengänge ist eine weitere Ausdifferenzierung und Diskussion des spezifischen Lehrgegenstands dieser verwandten Studiengänge zu erwarten und anzustreben. Eine Diskussion und Analyse der bisherigen Ergebnisse mit den Lehrverantwortlichen in der Versorgungsforschung ist wichtig. Auf diese Weise kann herausgefunden werden, wie die Fachbegriffe in den jeweiligen Studiengängen ausgelegt werden und welche Konzepte sich hinter den Zielen und Inhalten der Module der Versorgungsforschung verbergen. Um die Qualität des Studiengangsangebotes zur Versorgungsforschung auch in Zukunft zu sichern und um die Erwartungen der Adressaten und Anspruchsgruppen weiterhin zu erfüllen, wird ebenso zu diskutieren sein, inwieweit Empfehlungen für ein konsentiertes Kerncurriculum hierzu einen Beitrag leisten könnten.

\section{Interessenkonflikt}

Prof. Dr. Silke Kuske, Entwicklung und Studiengangsleitung des Masterstudiengangs Versorgungsforschung und Management im Gesundheitswesen M.Sc. an der Fliedner Fachhochschule Düsseldorf. Michel Wensing ist Studiengangsleiter des M.Sc. Programms Versorgungsforschung und Implementierungwissenschaft im Gesundheitswesen an der Universität Heidelberg. Prof. Dr. Bernd Reuschenbach ist Studiengangsleiter des Masterstudiengangs "Angewandte Versorgungsforschung“ an der Katholischen Stiftungshochschule München. Der in der Analyse aufgenommene Studiengang wird an der Katholischen Stiftungshochschule München angeboten. Prof. Dr. Andreas Büscher ist Studiengangsbeauftragter an der Hochschule Osnabrück für den Studiengang HELPP - Versorgungsforschung und -gestaltung. Prof. Dr. Katrin Balzer hat maßgeblich den Masterstudiengang Gesundheits- und Versorgungswissenschaften an der Universität zu Lübeck mitentwickelt und verantwortet den Vertiefungsschwerpunkt Versorgungsentwicklung und -management 
in diesem Studiengang. Prof. Dr. Sascha Köpke, bis 31.12.2020 Studiengangsleitung des M.Sc. Programms Gesundheits- und Versorgungswissenschaften an der Universität zu Lübeck. Prof. Dr. Holger Pfaff Studiengangsleitung des M.Sc. Programms Versorgungswissenschaft an der Universität zu Köln.

\section{Literatur}

[1] Blettner M, Dierks M-L, Donner-Banzhoff N et al. Überlegungen des Expertenbeirats zu Anträgen im Rahmen des Innovationsfonds. Z Evid Fortbild Qual Gesundhwes 2018; 130: 42-48. doi: 10.1016/j. zefq.2018.01.004

[2] Pfaff H, Neugebauer E, Glaeske G et al., Hrsg. Lehrbuch Versorgungsforschung: Systematik - Methodik - Anwendung. 2. Aufl. 2017

[3] Baumann W, Farin E, Menzel-Begemann A et al. Memorandum IV: Theoretische und normative Fundierung der Versorgungsforschung. Gesundheitswesen 2016; 78: 337-352. doi: 10.1055/s-0042-105511

[4] Ernstmann N, Heuser C, Pfaff H. Zur Situation der Versorgungsforschung an deutschen Universitäten, Hochschulen und Fachhochschulen. Gesundheitswesen 2020; 82: 313-317. doi: 10.1055/a-0668-5922

[5] Gruppe der Hochschullehrer des Deutschen Netzwerks für Versorgungsforschung. Positionspapier zur Situation der Versorgungsforschung an Universitäten, Hochschulen und Fachhochschulen in Deutschland. Im Internet: https://www.netzwerk-versorgungsforschung.de/index . php? page $=$ gruppe-der-hochschullehrerinnen Abruf 03.09.2020

[6] Glaeske G, Augustin M, Abholz H et al. Epidemiologische Methoden für die Versorgungsforschung. Gesundheitswesen 2009; 71: 685-693. doi: 10.1055/s-0029-1239517

[7] Icks A, Chernyak N, Bestehorn K et al. Methoden der gesundheitsökonomischen Evaluation in der Versorgungsforschung. Gesundheitswesen 2010; 72: 917-933. doi: 10.1055/s-0030-1262859

[8] Geraedts M, Drösler SE, Döbler K et al. DNVF-Memorandum III „Methoden für die Versorgungsforschung“, Teil 3: Methoden der Qualitäts- und Patientensicherheitsforschung. Gesundheitswesen 2017; 79: e95-e124. doi: 10.1055/s-0043-112431

[9] Koller M, Neugebauer EAM, Augustin M et al. Die Erfassung von Lebensqualität in der Versorgungsforschung - konzeptuelle, methodische und strukturelle Voraussetzungen. Gesundheitswesen 2009; 71: 864-872. doi: 10.1055/s-0029-1239516
[10] Stausberg J, Maier B, Bestehorn K et al. Memorandum Register für die Versorgungsforschung: Update 2019. Gesundheitswesen 2020; 82: e39-e66. doi: 10.1055/a-1083-6417

[11] Wirtz MA, Bitzer EM, Albert U-S et al. DNVF-Memorandum III - Methoden für die Versorgungsforschung, Teil 4 - Konzept und Methoden der organisationsbezogenen Versorgungsforschung. Kapitel 3 - Methodische Ansätze zur Evaluation und Implementierung komplexer Interventionen in Versorgungsorganisationen. Gesundheitswesen 2019; 81: e82-e91. doi: 10.1055/a-0862-0588

[12] Swart E, Gothe H, Geyer S et al. Gute Praxis Sekundärdatenanalyse (GPS): Leitlinien und Empfehlungen. Gesundheitswesen 2015; 77: 120-126. doi: 10.1055/s-0034-1396815

[13] Krippendorff K. Content analysis: An introduction to its methodology. 2014

[14] Eccles MP, Mittman BS. Welcome to implementation science. Implementation Sci 1, 1 (2006). https://doi.org/10.1186/1748-5908-1-1

[15] Ullrich C, Stürmlinger A, Wensing M. Wer braucht Versorgungsforscher/innen? Eine Bestandsaufnahme zum Berufsbild anhand von Stellenanzeigen. Z Evid Fortbild Qual Gesundhwes. 2019; 147-148 73-79. doi: 10.1016/j.zefq.2019.08.008

[16] Shortliffe EH, Garber AM. Training synergies between medical informatics and health services research: Successes and challenges. J Am Med Inform Assoc 2002; 9: 133-139. doi: 10.1197/jamia.m0974

[17] Scriba PC. Public Health und Versorgungsforschung: Herausforderungen zwischen Praxis und Politik. Gesundheitswesen 2009; 71: 606-609. doi: 10.1055/s-0029-1239568

[18] Badura B, Schaeffer D, Jv Troschke. Versorgungsforschung in Deutschland Fragestellungen und Förderbedarf. J Public Health 2001; 9: 294-311. doi: 10.1007/BF02956501

[19] Hülsken-Gießler Manfred, Brinker-Meyendriesch Elfriede, Keogh Johann et al. Kerncurriculum Pflegewissenschaft für pflegebezogene Studiengänge-eine Initiative zur Weiterentwicklung der hochschulischen Pflegebildung in Deutschland. Pflege \& Gesellschaft 2010; $15 \mathrm{Jg}$. H 3: 216-236

[20] DeBate R, Corvin JA, Wolfe-Quintero K et al. Application of the Intervention Mapping Framework to Develop an Integrated TwentyFirst Century Core Curriculum-Part 1: Mobilizing the Community to Revise the Masters of Public Health Core Competencies. Front Public Health 2017; 5: 287. doi: 10.3389/fpubh.2017.00287

[21] Corvin JA, DeBate R, Wolfe-Quintero K et al. Application of the Intervention Mapping Framework to Develop an Integrated Twentyfirst Century Core Curriculum-Part Two: Translation of MPH Core Competencies into an Integrated Theory-Based Core Curriculum. Front Public Health 2017; 5: 286. doi: 10.3389/fpubh.2017.00286 